\title{
Age differences in executive functioning across the lifespan: The role of verbalization in task preparation
}

\author{
Jutta Kray *, Jutta Eber, Ulman Lindenberger \\ Department of Psychology, Saarland University, Im Stadtwald, 66123 Saarbrücken, Germany
}

\begin{abstract}
Age-related changes in executive functioning across the lifespan were assessed in children (mean age $=9.4$ years), younger adults (mean age $=21.5$ years), and older adults (mean age $=65.3$ years). Executive functioning was investigated with a task-switching paradigm that permits the separation of two control components: to select and to switch between task sets. The specific aims of this study were (a) to determine developmental functions in both control components across the lifespan; and (b) to examine whether age-related changes in these components are influenced by verbal prompts during task preparation. The results revealed an inverted $\mathrm{u}$-shaped developmental function for the ability to select between task sets but not for the ability to switch between task sets. In contrast to younger adults and children, older adults generally benefited from verbalizations during task preparation. Children, but not older adults, showed a facilitation of task execution when verbal prompts were task-compatible. Conversely, older adults, but not children, showed stronger interference when verbal prompts are task-incompatible. Our findings suggest that inner speech in an important modulator of developmental changes in executive functioning across the lifespan.
\end{abstract}

(c) 2003 Elsevier B.V. All rights reserved.

PsycINFO classification: $2340 ; 2346 ; 2720 ; 2820$

Keywords: Executive functions; Aging; Lifespan; Task switching

\section{Introduction}

Empirical evidence from a number of studies indicates that age changes in executive functioning are closely linked to an increase in intellectual functioning during

\footnotetext{
${ }^{*}$ Corresponding author.

E-mail address: j.kray@mx.uni-saarland.de (J. Kray).
} 
childhood and a decrease in intellectual functioning in old age (e.g., Duncan, 1995). Executive functions generally refer to higher-level processes that organize lower-level processes in order to regulate and verify behavioral activity. Executive functioning is effective when it permits an individual to optimally adapt to continuous changes in the environment. Most researchers in the field now suggest that multiple functions or mechanisms are involved in the control of behavior (Goschke, 2000; Miyake et al., 2000; cf. also Gruber \& Goschke, 2004), or at least agree with current views on the kind of tasks and situations that require executive control (e.g., Shallice \& Burgess, 1993). Some researchers have argued that the ability to maintain goal-relevant information and to inhibit irrelevant information are among the most important functions of executive control, and that both of these functions are closely related to the integrity of prefrontal lobe functioning (e.g., Duncan, 1995; Miller \& Cohen, 2001; Roberts \& Pennington, 1996; see also Heyder, Suchan, \& Daum, 2004).

The aims of this paper are twofold: First, at a general level we will review some ideas how age-related changes in using verbal processes (such as inner speech) contribute to an understanding of age-related changes in executive functioning across the lifespan. Second, at a specific level we will report some findings from a first study that examined how age-related changes in executive control can be influenced by agerelated changes in using verbal prompts.

In the following we will first review evidence on age-related changes in executive functioning from a lifespan perspective. Then we will point out some theoretical ideas about the role of inner speech in the development of action regulation. Finally we will provide some empirical evidence how age-related changes in executive control components interact with age-related changes in using verbal prompts.

\subsection{Age differences in executive functioning across the lifespan}

In recent years a number of studies revealed an u-shaped or inverted u-shaped function (if performance is measured as costs) of age-related changes in tasks that are supposed to capture different aspects of executive functioning (cf. Zelazo, Craik, \& Booth, 2004) such as (a) the coordination of cognitive processing in complex tasks (e.g., Frensch, Lindenberger, \& Kray, 1999) and in figural transformations (e.g., Mayr, Kliegl, \& Krampe, 1996), (b) the inhibition of habitual response tendencies, such as in the Stroop Task (e.g., Comalli, Wapner, \& Werner, 1962), (c) the initiation and stopping of task execution (e.g., Bedard et al., 2002), and (d) the switching between task sets (e.g., Cepeda, Kramer, \& Gonzalez de Sather, 2001). U-shaped functions generally indicate an increase in intellectual functioning during childhood and a decrease in intellectual functioning during aging. Similar functions have been obtained for psychometric measures of fluid intelligence (e.g., Li et al., in press).

Optimally executive functioning requires that goal-directed actions are appropriately activated and maintained so that alternative action tendencies can be inhibited. According to this view, the inhibition of response tendencies is dependent on the optimal activation of working memory contents (cf. Roberts \& Pennington, 1996). Developmental researchers either emphasized the development of working memory span (e.g., Case, 1992), or the development of inhibitory processes (e.g., Dempster, 
1992), or the development of both processes (e.g., Diamond \& Taylor, 1996; Roberts \& Pennington, 1996) to be most important for the development of executive functioning.

Interestingly, the ability to maintain and manipulate task-relevant information as well as the ability to inhibit task-irrelevant information has also been attributed to prefrontal functioning, in general, and to dorsolateral prefrontal functioning, in particular (Milham et al., 2002; Miller \& Cohen, 2001). From an ontogenetic perspective it is important to note that the frontal lobes appear among the last regions of the brain to mature in childhood and adolescence, and among the first to show deterioration in old age. Therefore, several groups of investigators have proposed that agerelated changes in executive control are associated with age-related changes in the frontal lobes (Duncan, 1995; Prull, Gabrieli, \& Bunge, 2000; Raz, 2000; West, 1996; Zelazo et al., 2004).

\subsection{The role of inner speech in action regulation}

Only a few studies have focused on the question whether the ability to control one's own processing can be influenced by language, in general, and by inner speech, in particular (cf. Gruber \& Goschke, 2004; Miyake, Emerson, Padilla, \& Ahn, 2004). Inner speech refers to the activity of talking to oneself in silence, in contrast to talking to oneself aloud (private speech). The general function of inner or private speech is that of cognitive self-guidance (Vygotsky, 1988). In contrast to adults, especially younger children tend to talk to themselves aloud, which can be seen as an attempt to use language as a tool to plan, guide, and monitor goal-directed activity (Morin \& Everett, 1990).

Some decades ago, especially the Russian developmental psychologists (Luria, 1969; Meacham, 1979; Vygotsky, 1988) described how the development of the internalization of verbal processes becomes supportive for the development of action regulation between 2 and 7 years of age. For instance, according to Meacham (1979), the development of self-regulation through private speech runs through four stages. During the first stage, motor and verbal behavior may occur together but are independent of each other. During the second stage, the child begins to engage in verbal activity to describe the outcomes of his own motor behavior, verbalizations following motor actions. During the third stage, the child makes use of verbalizations to describe the anticipated goals of motor activity, the verbal activities thus may be subordinated. The important developmental transition involves not whether verbal activity follows or precedes motor activity, but rather whether verbal activity describes the outcomes of motor activity. In the fourth stage, verbalization in connection with other processes plays a major role in planning and guiding the course of motor behavior also termed "intellectualization of speech" (cf. Meacham, 1979).

According to Luria (1969), the inner speech system has a regulatory and planning function because "language is a signal of signals" that facilitates the identifying of goals, the relevant cues for orientation, and the organizing of temporal relations of motor activity such as if " $x$ then do $y$ ". The internalization of speech is also useful 
for guiding complex serial activity that requires the coordination of inhibitory as well as excitatory activity. During the internalization of speech we build verbal commands, mediated by socialization, that help to coordinate the serial order of inhibitory and excitatory activity. In other words such verbal commands can help to initiate or to stop our own actions or those of others.

Within current models of executive control, the role of inner speech is limited to the maintenance of information. For example, in the working memory model of Baddeley (1996) inner speech processes are closely related to the phonological loop system and in particular to one of its subcomponents the articulatory rehearsal process. The important function of this process is to actively maintain verbal/phonological information for further processing.

Whether inner speech has a more specific or a more general function in the control of cognitive activity is still an open question. The basic assumption of our research is that inner speech has a general function in action regulation in terms of focusing attention to goal-relevant knowledge. It may well be that inner speech helps to keep working memory contents in an optimal activation stage to prevent interference from other potentially relevant information. Hence, with the optimization of inner speech, age differences in executive functioning may become smaller. From an ontogenetic perspective the investigation of inner speech seems interesting for two reasons: First, as noted earlier the development of inner speech is especially important for learning, development, and regulation of action goals in childhood (Luria, 1969; Meacham, 1979; Morin \& Everett, 1990; Zelazo, 1999). Second, inner speech processes become well practiced during adulthood (cf. Emerson \& Miyake, 2003). Therefore, they may serve as a compensatory mechanism when executive abilities decrease in old age. Generally, our research interests concern the question whether age differences in executive functioning are modulated by inner speech. The goals of this study are much more specific and can be seen only as a first step to address this question.

\section{The present study}

The specific goals of the study are: (1) to introduce a paradigm that can be used to examine different aspects of executive functioning across the lifespan; (2) to examine whether the verbalization of task-related knowledge during task preparation produces costs or benefits, as compared with the (motor) execution of task-relevant knowledge, in switching between currently relevant tasks as well as age differences therein; and (3) to investigate whether verbalizations of task-compatible versus task-incompatible words can facilitate or inhibit task switching and whether age differences in these effects can be observed.

\subsection{The study of executive functioning via task switching}

Executive functioning has been frequently investigated by means of a task-switching paradigm (e.g., Rogers \& Monsell, 1995). Within that paradigm the participants 
are usually instructed to switch between two simple tasks A and B. For instance, in task A, they are instructed to decide whether the stimuli (e.g., different words) belong to the categories of animals or not. If it is an animal, then they should respond with the right response key; and if not, then they should respond with the left response key. In task B, they are asked to decide whether number of syllables is equal to one or two, and they are asked either to respond with the left or the right response button, respectively.

To measure the efficiency of executive control in task switching we determine performance in three different trial types: Under single-task conditions, participants perform the same task A or B throughout the whole block (i.e., in single-task trials); under mixed-task conditions, subjects are instructed to switch from one task to the other task (i.e., in switch trials, $\mathrm{AB}$ and $\mathrm{BA}$ trials) or to repeat the same task as in the preceding trial (i.e., in non-switch trials, AA and BB trials). The measurement of single and mixed-task performance allows the comparison between two types of switching costs. Switch costs are defined as the difference in performance on non-switch and switch trials within mixed-task blocks (e.g., Kramer, Hahn, \& Gopher, 1999; Meiran, 1996; Rogers \& Monsell, 1995). Another type of switching costs, termed set-selection costs, is defined as the difference in performance between single-task and mixed-task conditions (e.g., Kray \& Lindenberger, 2000; Mayr, 2001).

Although both components of control (indexed by selection and switch costs) have been demonstrated to be empirically independent, they are theoretically difficult to separate, because set switching also requires to keep two or more task sets in memory (cf. Brinley, 1965; Kray \& Lindenberger, 2000). The rationale for the investigation of age differences in task switching in our studies was to determine in a first step age differences related to the dual-task like situation, that is, to be in a switching situation which is associated with an increase in memory demands, and in a second step to examine whether age differences can additionally be found in the switch process per se. The disadvantage of this rationale is that the investigation of age differences in set-selection costs includes age differences in switch costs. Hence, if age differences in switch costs will be found it is necessary to determine set-selection costs without including the switch trials (see Kray, Li, \& Lindenberger, 2002).

In our previous research on adult age differences in task switching we usually found a pattern of greater age differences in set-selection costs than in switch costs (Kray, 2004a, 2004b; Kray \& Lindenberger, 2000; see also Mayr, 2001; but see De Jong, 2001; Meiran, Gotler, \& Perlman, 2001). So far only few studies have investigated age-related changes in executive functioning across the lifespan. Cepeda et al. (2001) used a task-switching paradigm to examine lifespan change in executive functioning from age 7 to 82 years. Results of their study indicated (a) that older adults and children show greater set-selection costs than younger adults; (b) that children show larger impairments in the ability to inhibit alternative response sets, compared with adults; and (c) that children and older adults show a greater reduction of setselection costs with increasing time to prepare the next task and with practice (see also Kray, 2004a). Important to note here is that these findings point to the fact that 
age-related changes in executive control components appear to be not unidirectional across the lifespan.

\subsection{Task switching and inner speech}

Similarly, only a few studies have attempted to specify the role of inner speech for goal-directed behavior such as in the control of switching between task sets (Baddeley, Chincotta, \& Adlam, 2001; Emerson \& Miyake, 2003; Goschke, 2000; Miyake et al., 2004). For instance, Goschke (2000) used a cue-based paradigm in which participants were instructed either to verbalize the task name or an irrelevant name when the time interval between cue and target (CTI) was very short or very long. Results of that experiment showed that switch costs were substantially reduced in the group instructed to verbalize the task name compared to the group that verbalize the irrelevant name but for long CTIs only.

In contrast to Goschke (2000), Emerson and Miyake (2003) used a list paradigm in which they could only determine performance in single-task blocks and mixedtask blocks (here termed set-selection costs; see also Baddeley et al., 2001). In this type of paradigm, the subjects are asked to switch between tasks via a predictable sequence. This condition requires participants to keep track of which task to perform next, hence maximizing the possibility of participants spontaneously recruiting inner speech. The most important finding of this study is that the disruption of inner speech in task switching was modulated by the presence and type of task-set cueing. The disruption of inner speech, by articulatory suppression, was maximal when external (task-set) cues are missing and internal cues had to be used. Under those conditions set-selection costs were substantially increased. In addition, Emerson and Miyake (2003) demonstrated that this result was not due to an increase in general dual-task demands because set-selection costs were not increased when the secondary task was a tapping task. The authors concluded that inner speech plays an important role as being an internal prompt that facilitates the retrieval of the phonological representation of the next task goal.

The result from both of these studies demonstrates that inner speech supports task-switching behavior. In the present study the focus was on whether age differences in task-switching costs are affected by verbalizations of task-relevant knowledge. To address this question we used a modified version of the task-switching paradigm as described earlier to determine two types of task switching costs, setselection costs and switch costs. A few changes in materials were needed to make the task-set switching paradigm amenable for use with children. Instead of using verbal materials, 32 different pictures of animals and fruits are presented.

In contrast to both previously described studies, the specific question of this experiment was to examine whether a differential activation of task knowledge at the level of response sets is possible. To investigate this we instructed our participants either to verbalize (read) task-compatible words, task-irrelevant words, or task-incompatible words during task preparation. These conditions are termed verbal secondary-task conditions. For instance, if the participants should perform the color task as indicated by the task-set cue "COLOR" (see Fig. 1), a task-compatible 


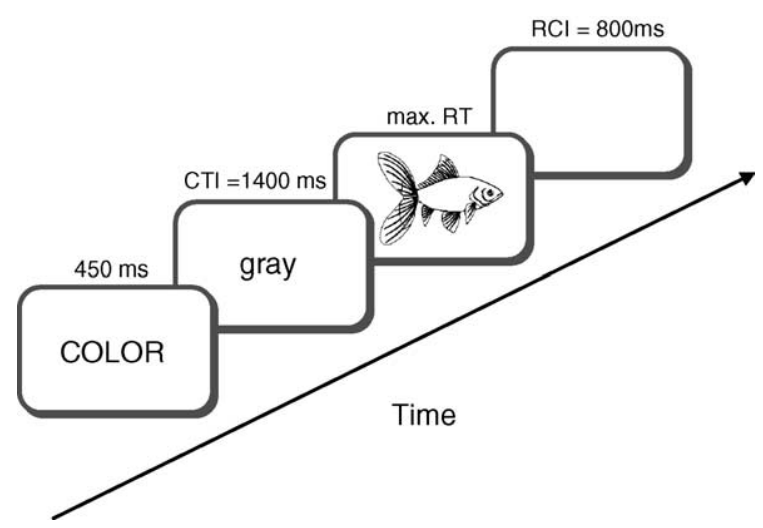

Fig. 1. Trial procedure. $\mathrm{CTI}=$ Cue-target interval. $\mathrm{RCI}=$ Response-cue interval.

word presented between cues and the target corresponds to the words "gray" or "colored", a task-irrelevant word to "sand" or "round", and a task-incompatible word to "animal" or "fruits". " To make sure that participants process this information during task preparation they were instructed to read the words aloud. To separate the effects of verbalization from a general increase in dual-task demands (cf. Emerson \& Miyake, 2003), we measured the performance in two control conditions. The first control condition was a no-secondary task condition in which the participants were instructed to do "nothing" during the preparation interval. The second control condition was a motor secondary task in which the participants were asked either to press the left response button when the sign "- --- " appears after the cue, or to press the right response button when the sign " ++++ " appears. This allows us to determine two types of dual-task demands: First, motor dual-task costs were specified as the difference in performance between the motor secondary-task condition and the no-secondary task condition; and verbal dual-task costs were defined as the difference between the verbal secondary-task condition and the no-secondary task condition.

To examine age differences in differential activation of task knowledge, two types of costs were defined: First, the difference in performance on task-compatible versus task-irrelevant words, termed facilitation effect; and second, the difference in performance on task-incompatible versus task-irrelevant words, termed interference effect.

To summarize the specific aims of this study: First, we modified the stimulus materials for studying task-switching behavior in middle childhood in order to investigate whether a similar pattern of age differences in set-selection costs and switch

\footnotetext{
${ }^{1}$ Note that in German all words were one-syllable words consisting of only four letters (cf., fruit [OBST], animal [TIER], gray [GRAU], colored [BUNT], Sand [SAND], and round [RUND]). We decided to use words as primes for two reasons: First, it is difficult to find prototypical pictures of these categories. Second, to separate perceptually the target during task preparation from the target during responding. To simplify the analysis of variance we only included trials that were also response compatible (for taskcompatible conditions) or response incompatible (for task-incompatible conditions).
} 
costs as observed in older adults can also be found in children. The second aim is to examine whether age-related differences in task-switching components are enhanced or reduced as a function of the nature of dual-task demands (i.e., verbal versus motor secondary task) in task preparation. Our third aim is to explore whether age differences in facilitation and interference effects can be obtained depending on verbalizations of task-compatible versus task-incompatible words during task preparation.

\section{Method}

\subsection{Participants}

Fifty-two participants were recruited for a two-session experiment. Four children were not able to perform the experimental task, so that they did not participate in the second session and were excluded from data analysis. The final sample includes 16 young adults (mean age $=21.5$ years, $\mathrm{SD}=1.6$; age range $=20-25$ years of age, $50 \%$ female), 16 older adults (mean age $=65.3$ years $\mathrm{SD}=3.8$; age range $=61-72$ years of age, $50 \%$ female), and 16 children (mean age $=9.4$ years, $\mathrm{SD}=0.7$; age range $=8-10$ years of age, $50 \%$ female). All participants were paid 12 Euro (about $\$ 12$ US) for participating.

Two psychometric tests, one from the fluid domain and one from the crystallized domain of intelligence, were used to indicate the typically of the sample. As expected on the basis of the literature, we found highly reliable age differences on a test of perceptual speed, the Digit-Symbol Substitution Test, ${ }^{2} F(2,45)=45.13$, $\mathrm{MSE}=3292.19, p<0.01$. Younger adults $(M=62.6, \mathrm{SD}=9.01)$ reached significantly higher scores than older adults $(M=44.3, \mathrm{SD}=9.7 ; t(1,45)=6.08$, $p<0.01)$, and older adults scored higher than children $(M=34.3, \mathrm{SD}=6.6$; $t(1,45)=3.29, p<0.01)$.

We also observed significant age differences on a vocabulary subtest, ${ }^{3}$ $F(2,45)=62.27, p<0.01, \mathrm{MSE}=640.13, p<0.01$. Children obtained a lower score $(M=13.1, \mathrm{SD}=4.5)$ in the vocabulary subtest than younger adults $(M=23.8$, $\mathrm{SD}=3.3 ; \quad t(1,45)=-2.72, \quad p<0.01) ; \quad$ in contrast, older adults $(M=27.4$, $\mathrm{SD}=3.4)$ obtained significantly higher scores than younger adults, $t(1,45)=$ $10.73, p<0.01$.

\footnotetext{
${ }^{2}$ An adapted version of the Wechsler Digit-Symbol Substitution Test was used to measure perceptual speed of processing. The test sheet was enlarged to reduce problems related to poor vision or motor abilities. The template contained nine digit-symbol mappings. The participants were instructed to fill in the symbol that corresponds to the digit. The score was the number of correct symbols after $90 \mathrm{~s}$.

${ }^{3}$ In this test, 35 items are presented successively on the screen. Each item contained one correct word and four non-words. The participants are asked to find the one correct word. The score was the number of correct words.
} 


\subsection{Materials, stimuli, and tasks}

We used IBM-compatible computers for data collection. The stimuli were presented on a CTX 17-inch color monitor with a black background. The stimulus set consisted of 32 pictures, 8 animals and 8 fruits. Half of the pictures was gray and the other half was colored. In the one task, participants were asked to decide whether the objects belong to the category of animals (e.g., fish, horse, butterfly, and so on) or fruits (e.g., melon, orange, strawberry, and so on). In the second task they had to decide whether the color of these pictures was gray or colored. Subjects were instructed to press the right response key if the picture was an animal or colored and otherwise the left key. We used highly overlapping task-set representations meaning that attributes of both tasks were activated at the same time and attributes were in half of the cases mapped onto the same response button (cf. Rogers \& Monsell, 1995).

Whether the object or the color task had to be performed was indicated by semantic instructional cues ("OBJECT" for the object task and "COLOR" for the color task; see Fig. 1). The cues and targets were displayed in uppercase 34-bitmap font (Swiss regular).

To measure age differences in the use of verbal prompts as well as the effects of facilitation and inhibition by reading task-compatible and task-incompatible words during the task preparation the following experimental conditions were varied. The participants were instructed (1) either to read the words (fruits, animals, gray, colored, sand, round) that were presented after the instructional cues and remained until the target was presented, (2) to press one of the response keys when there were symbols instead of words (press the left key if the letter string " --- -" appears; press the right key if the letter string " ++++ " appears) or (3) to "do nothing" if a star $\left(^{*}\right)$ was presented. All conditions were varied within blocks. Because the experimental task was quite difficult, we investigated a subsample of subjects that already participated in a previous experiment ${ }^{4}$ (Kray, 2004a), so that all participants were practiced in the stimulus-response assignments. In the previous experiment the same two task sets (color and object tasks) were used but without additionally secondary task demands, that is, the experimental conditions in the previous experiment correspond to the no secondary-task condition in the present experiment.

\subsection{Procedure}

The two experimental sessions lasted approximately $45 \mathrm{~min}$. All participants were tested individually. During the experimental phase, all participants had a short break after about 25 min of testing.

Both sessions began with a practice phase consisting of two blocks of each single task, the object and the color task and two mixed-task blocks. In the first session,

\footnotetext{
${ }^{4}$ Information about demographic variables, the Digit-Symbol Substitution test, and the Vocabulary subtest was taken from a previous experiment that was carried out a few months prior to the present study.
} 
two short blocks including 9 trials were given in order to demonstrate all task conditions. Altogether the participants worked through 16 blocks, 8 single-task blocks and 8 mixed-task blocks. The sequence of experimental blocks was random with the constraint that the two single-task blocks (object, color) and two mixed-task blocks were blocked together (i.e., 4 miniblocks). The order of single and mixed-task blocks within these miniblocks was random as well as the sequence of the miniblocks across the experiment. The sequence of single and mixed-task blocks was constant across subjects and experimental conditions while the sequence of trials within each block was varied across subjects. The experimental procedure of the second session was identical to the first experimental session except that the practice phase only included the two mixed-task blocks.

Each block consisted of 37 trials, yielding a total of 2 sessions $\times 16$ blocks $\times 37$ trials $=1184$ trials. The first trial in each block was not analyzed; this trial is drawn from a pool of practice stimuli. The single and mixed blocks consisted of an equal number of the four stimulus types (fruit/gray, fruits/colored, animals/gray, animals/colored), and secondary task instructions: read the task-relevant words (fruits, animals, gray, colored) or irrelevant words (sand, round), press a response key (left, right), and "do nothing".

We used the following trial procedure (see Fig. 1): Each trial started with an instructional cue (i.e., object, color) that remained for $450 \mathrm{~ms}$, followed by a fixed cue-target interval (CTI) of $1400 \mathrm{~ms}$. Immediately after the instructional cue disappeared, one of the additional task instructions (words or symbols) were presented and remained until the target was presented. The target remained until the response was made. The time between the response and the next instructional cue (RCI) was fixed to $800 \mathrm{~ms}$ to avoid carryover effects from the preceding trial.

Both verbal and visual general instructions to the experimental tasks were provided. Participants were told to respond as quickly and as accurately as possible. Before each block an instruction window appeared that indicated whether the object or the color task, or both tasks have to be performed in the following block. After each experimental block, feedback regarding the subject's mean response times and percentage of errors was given. Secondary task errors, such as when the participants responded to early or to late, or did not press the response in the CTI were documented separately in written protocols by the experimenter.

\section{Results and discussion}

Analysis of variance (ANOVA) was based on mean reaction times (RTs) for correct responses or on error rates. All trials in which the participants made a response error or a secondary task error, such as not reading the word aloud or pressing the button in the preparation interval, were excluded from data analysis. In addition, all responses faster than $180 \mathrm{~ms}(0.54 \%$ for the children, $0.09 \%$ for the younger adults; and $0.29 \%$ for the older adults) and slower than $3500 \mathrm{~ms}$ (3.11\% for the children, $0.21 \%$ for the younger adults; and $0.52 \%$ for the older adults) were excluded from data analysis. 
To control for age differences in baseline performance, a logarithmic transformation of RTs was used. The advantage of this method is that the difference between log-transformed RTs of two conditions corresponds to proportional scores. Hence, age-by-condition interactions were interpreted on the basis of proportional scores and not on the basis of difference scores (cf. Meiran, 1996). All results for log-transformed RTs were significant at $p<0.01$, unless specified otherwise. In the analysis of variance, Age was the between-subjects factor and Trial Type (single, non-switch, switch) and Secondary Task Condition (no, motor, task-compatible, task-incompatible, task irrelevant) were the within-subjects factors.

The overall ANOVA indicated significant main effects of Age, $F(2,45)=42.45$, $\mathrm{MSE}=0.658, p<0.01$, Trial Type, $F(2,90)=130.49$, MSE $=0.011, p<0.01$, and Secondary Task Condition, $F(4,180)=20.30, \mathrm{MSE}=0.014, p<0.01$ as well as significant interactions of Age $\times$ Trial Type, $F(4,90)=10.38, \mathrm{MSE}=0.011, p<0.01$, Age $\times$ Secondary Task Condition, $F(8,180)=5.24, \mathrm{MSE}=0.014, p<0.01$, and Trial Type $\times$ Secondary Task Condition, $F(8,360)=8.31, \mathrm{MSE}=0.004, p<0.01$. In the following we will only test a-priori contrasts that are reported in a stepwise fashion along the main goals of the present study.

\subsection{Age differences in set-selection and switch costs}

To determine age differences in task switching, the factor Trial Type was specified as two orthogonal, within-subjects contrasts. The first contrast was defined as the difference in performance between single-task trials and mixed-task trials (set-selection costs), and the second contrast was defined as the difference in performance between non-switch and switch trials (switch costs).

The analysis of latencies indicated highly reliable age differences in set-selection costs, $F(2,45)=13.88$, MSE $=0.474, p<0.01$, but no reliable age differences in switch costs $(p=0.41)$. As shown in Fig. 2, children showed substantially greater set-selection costs than older adults, $F(1,45)=11.37, \mathrm{MSE}=0.474, p<0.01$, and younger adults, $F(1,45)=26.96, \mathrm{MSE}=0.474, p<0.01$. Set-selection costs were also significantly greater for older than for younger adults at the 5\%-level, $F(1,45)=3.95, \mathrm{MSE}=0.474, p=0.05$. Furthermore, both u-shaped and linear age trends in set-selection costs were highly reliable, $F(1,45)=16.40, \mathrm{MSE}=0.474$, $p<0.01 ; F(1,45)=11.37, \mathrm{MSE}=0.474, p<0.01$.

Similarly, the analysis of error rates showed significant age differences in set-selection costs, indicating higher error rates in mixed-task blocks than in single-task blocks for older than for younger adults, $F(1,45)=12.31, \mathrm{MSE}=772.70$, $p<0.01$, and for children than for younger adults, $F(1,45)=9.72$, MSE $=772.70$, $p<0.01$. In addition, older adults made significantly more errors than younger adults on switch trials than on non-switch trials, that is, they showed greater error switch costs $F(1,45)=6.01, \mathrm{MSE}=149.39, p=0.02$.

The first major result of the present study is that children, like older adults, show large performance deficits in set selection compared to young adults but less in switching per se. 

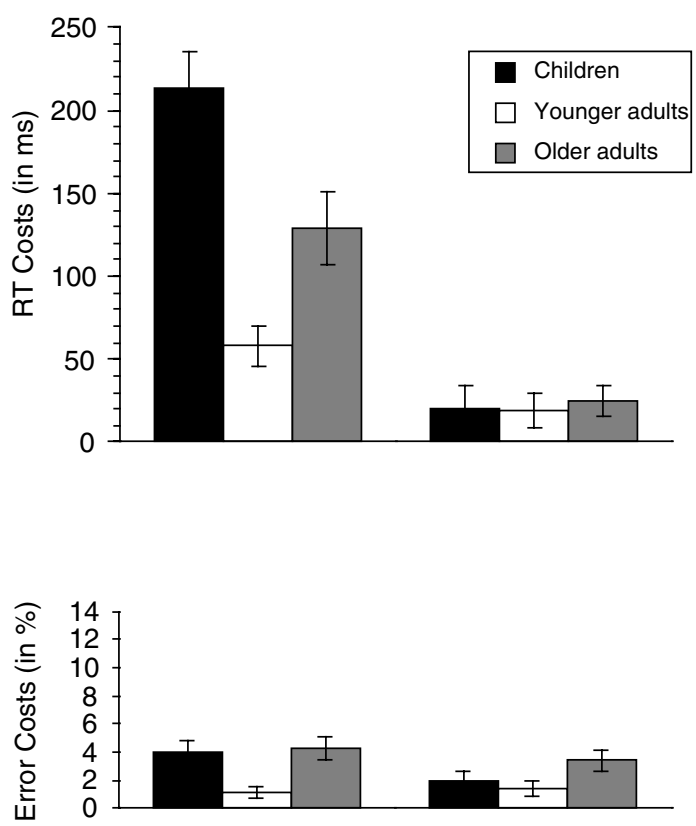

Fig. 2. Set-selection costs (on the left) and switch costs (on the right) as a function of age group (children, young, old). RT costs (in ms) are displayed at the top and error costs (in \%) at the bottom. Error bars refer to the standard errors.

\subsection{Age differences in performing a secondary task during task preparation}

Age differences in costs or benefits of performing a secondary task were specified by the following contrasts in the ANOVA. First, costs of performing a motor secondary task were determined as the difference in performance between motor-task trials and no-secondary task trials (motor dual-task costs); costs of performing a verbal secondary task were determined as the difference in performance between the mean of all verbalization trials and no-secondary task trials (verbal dual-task costs); third, the difference between the type of dual-task demands was tested as the difference in performance between motor-task trials and verbalization trials (generality of dual-task demands).

Table 1 shows mean RTs as a function of age, the type of the secondary task condition, and trial type. The analysis of latencies revealed highly significant motor dual-task costs in all three age groups, suggesting that latencies were significantly slowed when a motor secondary task has to be performed during task preparation, $F(2,45)=43.97, \mathrm{MSE}=0.111, p<0.01$, but age differences in motor dual-task costs were only significant on the basis of difference scores $(p<0.05)$, not on the basis of proportional scores (on the basis of log-transformed RTs). In addition, highly reliable age differences in verbal dual-task costs were found, $F(2,45)=6.08$, $\mathrm{MSE}=0.782, p<0.01$. Children and younger adults showed greater verbal dual- 
Table 1

Mean reaction times (ms) and SDs for children, younger and older adults as a function of trial type, and secondary task type

\begin{tabular}{|c|c|c|c|c|c|c|}
\hline \multirow[t]{2}{*}{ Variable } & \multicolumn{2}{|c|}{ Children } & \multicolumn{2}{|c|}{ Younger adults } & \multicolumn{2}{|c|}{ Older adults } \\
\hline & $M$ & SD & $M$ & SD & $M$ & SD \\
\hline \multicolumn{7}{|c|}{ No-secondary task } \\
\hline Single & 888 & 200 & 488 & 145 & 760 & 156 \\
\hline Mixed & 1041 & 239 & 507 & 160 & 871 & 219 \\
\hline Non-switch & 1062 & 244 & 509 & 137 & 883 & 233 \\
\hline Switch & 1021 & 248 & 505 & 186 & 859 & 216 \\
\hline \multicolumn{7}{|c|}{ Motor secondary task } \\
\hline Single & 962 & 182 & 517 & 156 & 853 & 236 \\
\hline Mixed & 1226 & 244 & 561 & 176 & 1030 & 307 \\
\hline Non-switch & 1223 & 234 & 569 & 191 & 1022 & 313 \\
\hline Switch & 1228 & 269 & 553 & 165 & 1038 & 305 \\
\hline \multicolumn{7}{|c|}{ Verbal secondary task } \\
\hline Single & 908 & 200 & 480 & 131 & 715 & 123 \\
\hline Mixed & 1140 & 230 & 551 & 185 & 827 & 177 \\
\hline Non-switch & 1116 & 212 & 534 & 162 & 805 & 179 \\
\hline Switch & 1164 & 256 & 568 & 211 & 849 & 178 \\
\hline
\end{tabular}

task costs than older adults, $\quad F(1,45)=11.05, \quad \mathrm{MSE}=0.782, \quad p<0.01$; $F(1,45)=6.61, \mathrm{MSE}=0.782, p=0.01$. Testing for u-shaped and linear age trends indicated only a significantly linear age trend for verbal dual-task costs, $F(1,45)=11.05, \mathrm{MSE}=0.782, p<0.01$.

The analysis of variance further indicated that dual-task costs were greater in the motor secondary task than in the verbal secondary task, $F(1,45)=36.24$, $\mathrm{MSE}=0.911, p<0.01$. The effect was even more pronounced for older adults than for younger adults and children, $F(1,45)=14.84, \quad \mathrm{MSE}=0.911, \quad p<0.01$; $F(1,45)=7.93, \mathrm{MSE}=0.911, p<0.01$, but no reliable difference between younger adults and children was obtained. Both u-shaped and linear age trends were highly reliable, $F(1,45)=7.96, \mathrm{MSE}=0.911, p<0.01 ; F(1,45)=7.93, \quad \mathrm{MSE}=0.911$, $p<0.01$.

The corresponding error rates are displayed in Table 2. In line with the results on latencies, the analysis of error rates revealed that participants made more errors when either a motor task or a verbal task had to be performed during task preparation, $F(1,45)=19.51, \mathrm{MSE}=386.74, p<0.01 ; F(1,45)=37.73, \mathrm{MSE}=62.47$, $p<0.01$, but no reliable differences in error dual-task costs were observed between children, younger, and older adults, neither for the motor nor for the verbal secondary task.

\subsubsection{Interactions with set-selection costs}

In all three age groups, a significant increase in motor dual-task costs was found for mixed-task blocks as compared to single-task blocks, $F(1,45)=11.24$, $\mathrm{MSE}=0.040, p<0.01$, but no reliable interactions with age were observed. A signif- 
Table 2

Error rates (\%) and SDs for children, younger and older adults as a function of trial type, and secondary task type

\begin{tabular}{|c|c|c|c|c|c|c|}
\hline \multirow[t]{2}{*}{ Variable } & \multicolumn{2}{|c|}{ Children } & \multicolumn{2}{|c|}{ Younger adults } & \multicolumn{2}{|c|}{ Older adults } \\
\hline & $M$ & SD & $M$ & SD & $M$ & SD \\
\hline \multicolumn{7}{|c|}{ No-secondary task } \\
\hline Single & 4.87 & 4.75 & 2.57 & 2.17 & 1.08 & 1.97 \\
\hline Mixed & 7.08 & 4.66 & 1.67 & 1.76 & 5.50 & 3.67 \\
\hline Non-switch & 7.86 & 8.02 & 2.16 & 2.73 & 6.78 & 4.76 \\
\hline Switch & 6.31 & 3.70 & 1.18 & 1.95 & 4.22 & 3.71 \\
\hline \multicolumn{7}{|c|}{ Motor secondary task } \\
\hline Single & 5.26 & 3.40 & 3.31 & 3.31 & 1.98 & 1.35 \\
\hline Mixed & 9.86 & 7.61 & 4.18 & 3.73 & 6.77 & 4.69 \\
\hline Non-switch & 12.13 & 9.68 & 4.32 & 4.60 & 5.60 & 5.33 \\
\hline Switch & 7.58 & 6.04 & 4.04 & 3.28 & 7.95 & 5.08 \\
\hline \multicolumn{7}{|c|}{ Verbal secondary task } \\
\hline Single & 5.61 & 4.17 & 3.82 & 2.45 & 2.48 & 2.87 \\
\hline Mixed & 10.32 & 5.23 & 5.11 & 4.40 & 7.61 & 5.23 \\
\hline Non-switch & 7.04 & 4.29 & 4.12 & 3.04 & 3.77 & 2.63 \\
\hline Switch & 13.60 & 6.76 & 6.10 & 6.09 & 11.45 & 8.47 \\
\hline
\end{tabular}

icant increase in verbal dual-task costs was also observed under mixed versus singletask conditions, $F(1,45)=11.42$, MSE $=0.040, p<0.01$.

In addition, the analysis revealed a reliable interaction between the type of dualtask costs, set-selection costs, and age, $F(2,45)=3.50, \mathrm{MSE}=0.245, p=0.04$ (see Fig. 3, at the top). The increase in dual-task costs for mixed-task blocks was greater for older adults than for younger adults when the secondary task condition was a motor and not a verbal task, $F(1,45)=6.96, \mathrm{MSE}=0.245, p=0.01$.

The analysis of errors only indicated a significant increase in verbal dual-task costs for mixed-task blocks relative to single-task blocks, $F(1,45)=9.17$, $\mathrm{MSE}=319.31, p<0.01$. This increase in costs was found to be less pronounced for older adults than for younger adults and children, $F(1,45)=6.33$, $\mathrm{MSE}=319.31, p=0.02, F(1,45)=5.08, \mathrm{MSE}=319.31, p=0.03$.

\subsubsection{Interactions with switch costs}

The analysis of latencies indicated that switch costs were significantly increased in the verbal secondary task as compared with the no-secondary task, $F(1,45)=26.46$, MSE $=0.099, p<0.01$, but not for the motor secondary task (see Fig. 3, at the bottom). The increase in switch costs was more pronounced in the verbal than in the motor secondary task, $F(1,45)=12.90, \mathrm{MSE}=0.115, p<0.01$. However, no significant interactions between dual-task demands, switch costs, and age were obtained.

The analysis of errors indicated that participants made more errors in switch than in non-switch trials when the secondary task was verbal, $F(1,45)=29.81$, $\mathrm{MSE}=515.22, p<0.01$, and that the increase in error rate on switch trials compared with non-switch trials was more pronounced in the verbal than in the motor task, 

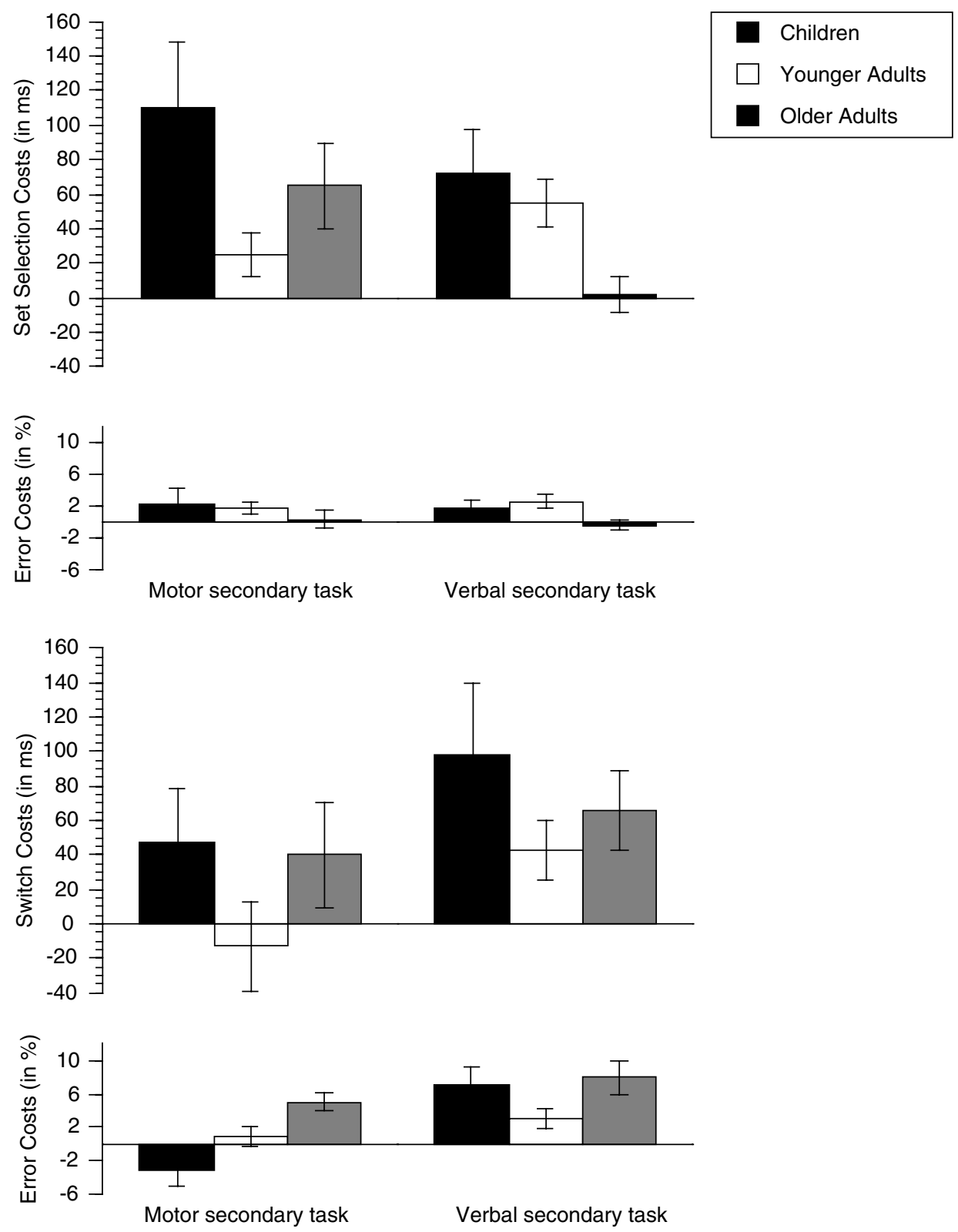

Fig. 3. Dual-task costs as a function of type of secondary task (motor, verbal), and age group (children, young, old). Set-selection costs (RT and error costs) are displayed at the top; switch costs (RT and error costs) at the bottom. Error bars refer to the standard errors.

$F(1,45)=34.57, \mathrm{MSE}=324.37, p<0.01$. Fig. 3 also shows that this effect was greater in children as compared with older adults and younger adults, 
$F(1,45)=9.11, \quad \mathrm{MSE}=324.37, \quad p<0.01 ; \quad F(1,45)=10.75, \quad \mathrm{MSE}=324.37, \quad p<$ 0.01 .

In sum, as can be seen in Fig. 3, the results on age differences in latencies are generally consistent with the results on age differences in error rates indicating that the findings are not confounded by age differences in speed-accuracy trade-offs.

\subsection{Age differences in priming of response sets during task preparation}

To determine age differences in facilitation and interference effects, the following contrasts were specified. Facilitation effects are defined as the difference in performance between task-compatible and task-irrelevant trials (thus facilitation is indicated by negative values); and interference effects are specified as the difference in performance between task-incompatible and task-irrelevant trials (interference is indicated by positive values).

The analysis of latencies revealed reliable facilitation effects, $F(1,45)=6.58$, $\mathrm{MSE}=0.036, p=0.01$, indicating that latencies were faster when subjects verbalize task-compatible words compared with task-irrelevant words (see Table 3). The facilitation effect was even more pronounced for children than for older adults, $F(1,45)=4.65, \mathrm{MSE}=0.036, p=0.04$; and for younger than for older adults, $F(1,45)=4.41, \mathrm{MSE}=0.036, p=0.04$. In contrast, substantially greater interference effects were found for older adults than for children, $F(1,45)=6.18$, MSE $=0.029, p=0.02$, and for older adults than for younger adults, $F(1,45)=4.00, \mathrm{MSE}=0.029, p=0.05$. Testing for $\mathrm{u}$-shaped and linear age trends revealed that only linear age trends were reliable for both, the facilitation effect,

Table 3

Mean reaction times (ms) and SDs for children, younger and older adults as a function of trial type, and secondary verbalization task

\begin{tabular}{|c|c|c|c|c|c|c|}
\hline \multirow[t]{2}{*}{ Variable } & \multicolumn{2}{|c|}{ Children } & \multicolumn{2}{|c|}{ Younger adults } & \multicolumn{2}{|c|}{ Older adults } \\
\hline & $M$ & SD & $M$ & SD & $M$ & SD \\
\hline \multicolumn{7}{|c|}{ Task-compatible verbalization } \\
\hline Single & 865 & 178 & 460 & 114 & 699 & 122 \\
\hline Mixed & 1120 & 228 & 532 & 188 & 833 & 197 \\
\hline Non-switch & 1148 & 230 & 522 & 160 & 817 & 203 \\
\hline Switch & 1092 & 255 & 542 & 222 & 849 & 200 \\
\hline \multicolumn{7}{|c|}{ Task-incompatible verbalization } \\
\hline Single & 919 & 224 & 498 & 156 & 745 & 136 \\
\hline Mixed & 1147 & 250 & 562 & 188 & 839 & 187 \\
\hline Non-switch & 1087 & 228 & 551 & 160 & 824 & 182 \\
\hline Switch & 1206 & 314 & 574 & 223 & 855 & 200 \\
\hline \multicolumn{7}{|c|}{ Task-irrelevant verbalization } \\
\hline Single & 940 & 214 & 483 & 125 & 700 & 117 \\
\hline Mixed & 1153 & 254 & 557 & 188 & 810 & 162 \\
\hline Non-switch & 1114 & 241 & 528 & 173 & 776 & 172 \\
\hline Switch & 1193 & 292 & 586 & 209 & 844 & 157 \\
\hline
\end{tabular}


Table 4

Error rates (\%) and SDs for children, younger and older adults as a function of trial type, and secondary verbalization task

\begin{tabular}{|c|c|c|c|c|c|c|}
\hline \multirow[t]{2}{*}{ Variable } & \multicolumn{2}{|c|}{ Children } & \multicolumn{2}{|c|}{ Younger adults } & \multicolumn{2}{|c|}{ Older adults } \\
\hline & $M$ & SD & $M$ & $\mathrm{SD}$ & $M$ & SD \\
\hline \multicolumn{7}{|c|}{ Task-compatible verbalization } \\
\hline Single & 5.18 & 4.53 & 3.65 & 3.05 & 1.57 & 2.80 \\
\hline Mixed & 8.68 & 4.55 & 5.12 & 4.75 & 4.86 & 4.73 \\
\hline Non-switch & 9.36 & 4.32 & 5.23 & 4.63 & 2.32 & 3.04 \\
\hline Switch & 8.01 & 6.58 & 5.02 & 7.19 & 7.39 & 7.56 \\
\hline \multicolumn{7}{|c|}{ Task-incompatible verbalization } \\
\hline Single & 6.65 & 5.59 & 5.01 & 3.97 & 3.16 & 3.77 \\
\hline Mixed & 13.45 & 6.53 & 5.17 & 4.86 & 12.44 & 9.76 \\
\hline Non-switch & 5.80 & 4.62 & 3.96 & 3.78 & 6.23 & 5.13 \\
\hline Switch & 21.10 & 10.34 & 6.38 & 7.06 & 18.65 & 15.53 \\
\hline \multicolumn{7}{|c|}{ Task-irrelevant verbalization } \\
\hline Single & 5.00 & 3.83 & 2.79 & 2.50 & 2.70 & 3.48 \\
\hline Mixed & 8.82 & 5.92 & 5.04 & 4.72 & 5.54 & 3.75 \\
\hline Non-switch & 5.96 & 6.62 & 3.17 & 3.29 & 2.76 & 2.83 \\
\hline Switch & 11.69 & 6.68 & 6.90 & 6.62 & 8.32 & 5.44 \\
\hline
\end{tabular}

$F(1,45)=4.65, \mathrm{MSE}=0.036, p=0.04$, and the interference effect, $F(1,45)=6.18$, $\mathrm{MSE}=0.029, p=0.02$.

The analysis of errors (see Table 4) only indicated a reliable interference effect, that is, participants made more errors on task-incompatible than on task-irrelevant trials, $F(1,45)=30.17$, MSE $=135.20, p<0.01$. This effect was even more pronounced for older adults than for children, $F(1,45)=8.17$, MSE $=135.20$, $p<0.01$; and for children than for younger adults, $F(1,45)=4.19$, MSE $=135.20$, $p=0.05$.

\subsubsection{Interactions with set-selection costs}

Set-selection costs increased when subjects verbalize task-compatible words compared with task-irrelevant words, $F(1,45)=7.17, \mathrm{MSE}=0.024, p=0.01$, whereas age differences in set-selection costs were not modulated by facilitation effects.

In contrast to the analysis of latencies, error rates were substantially higher on task-incompatible than on task-irrelevant trials for mixed-task blocks than for single-task blocks, $F(1,45)=9.22, \mathrm{MSE}=124.11, p<0.01$. Moreover, this effect was greater for children than younger adults, $F(1,45)=18.75, \mathrm{MSE}=124.11, p<0.01$, and for older than for younger adults, $F(1,45)=6.60, \mathrm{MSE}=124.11, p=0.01$. Thus, age differences in set-selection costs (on the basis of response errors) were enlarged by processing of task-incompatible information during task preparation.

\subsubsection{Interactions with switch costs}

Similar to set-selection costs, switch costs were significantly increased for taskcompatible than task-irrelevant trials, $F(1,45)=7.10, \mathrm{MSE}=0.025, p=0.01$. 
Again, age differences in switch costs were not affected by facilitation or interference effects.

Consistent with the analysis of latencies, participants made more errors in switch than in non-switch trials when they verbalize task-irrelevant compared with taskcompatible words, $F(1,45)=11.65, \mathrm{MSE}=60.71, p<0.01$. This effect was larger for children than for older adults, $F(1,45)=5.72, \mathrm{MSE}=60.71, p=0.02$ (see Table 4). In addition, error rates were substantially higher in switch than in nonswitch trials, $F(1,45)=15.27, \mathrm{MSE}=79.83, p<0.01$, when participants verbalize

Children
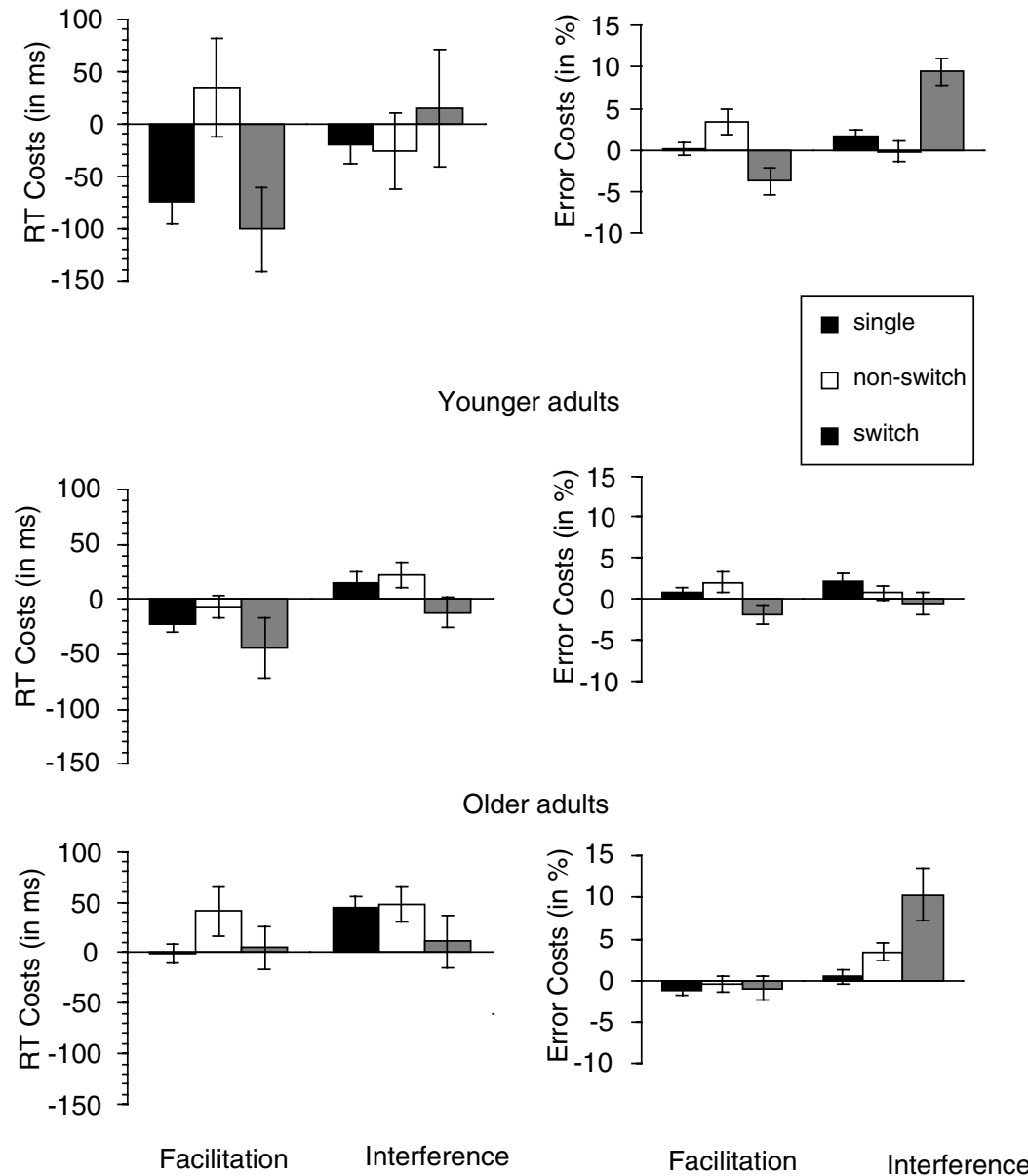

Fig. 4. Facilitation (task-compatible-task-irrelevant verbalization) and interference (task-incompatible - task irrelevant verbalization) effects as a function of trial type (single, non-switch, switch) and age group (children, young, old). RT costs (in ms) are displayed at the left side and error costs (in \%) at the right side. Error bars refer to the standard errors. 
task-incompatible compared with task-irrelevant words. Again, this effect was greater for children than for younger adults, $F(1,45)=18.75$, MSE $=124.11$, $p<0.01 ; F(1,45)=6.72$, MSE $=79.83, p=0.01$, and for older than for younger adults, $F(1,45)=6.60, \mathrm{MSE}=124.11, p=.01 ; F(1,45)=11.89, \quad \mathrm{MSE}=79.83$, $p<0.01$.

\subsubsection{Separate analysis of trial types}

Separate analysis of single, non-switch and switch trials revealed a couple of interesting age differences in facilitation and interference effects. Fig. 4 displays facilitation and interference effects as a function of task-compatible and task-incompatible verbalizations and trial type separately for children (at the top), young adults (in the middle), and older adults (at the bottom). Mean latencies are shown on the left side and percentages of errors on the right side.

In single-task trials, a highly reliable facilitation effect was found, $F(1,45)=21.57, \mathrm{MSE}=0.004, p<0.01$. This effect was significantly larger for children than for older adults, $F(1,45)=8.67, \mathrm{MSE}=0.004, p<0.01$. Interference effects were not reliable in single-task trials but significant age differences in interference effects were obtained. As can be seen in Fig. 4, older adults showed greater interference effects as compared with children, $F(1,45)=17.32, \mathrm{MSE}=0.003$, $p<0.01$, and with younger adults, $F(1,45)=4.89, \mathrm{MSE}=0.003, p=0.03$.

In non-switch trials, older adults also showed larger interference effects than children, $F(1,45)=4.32$, MSE $=0.007, p=0.04$. In contrast, in switch trials highly reliable facilitation effects can be found, $F(1,45)=7.62$, MSE $=0.012, p<0.01$, but no reliable interference effects. The facilitation effect was again larger for children than for older adults, $F(1,45)=4.28, \mathrm{MSE}=0.012, p=0.04$.

In sum, it appears that older adults have major deficits in inhibiting task-incompatible information during task preparation. Children, in contrast, show primarily benefits from verbalization of task-compatible words.

\section{Discussion}

In this study we assessed age-related changes in executive functioning across the lifespan by means of a cue-based task-switching paradigm. The primary goal was to examine whether age differences in set selection (set-selection costs) and set switching (switch costs) were affected when task preparation was systematically manipulated by different types of secondary task demands, verbal and motor tasks, that were either compatible or incompatible with the currently relevant task.

So far only a few studies have examined age differences in task switching across the lifespan (cf. Cepeda et al., 2001). Hence, the first important result to note is that children, like older adults, showed much greater set-selection costs than younger adults, whereas no age differences in switch costs can be obtained. The present results join others in demonstrating that age functions in executive control components are not unidirectional across the lifespan (cf. Cepeda et al., 2001; see also Zelazo et al., 
2004). In this study an inverted u-shaped age function can be found for the ability to select between task sets but not for the ability to switch between them.

The specific goal of the present study was to examine the role of inner speech in task-switching behavior. The few studies that have investigated the disruption of inner speech in task switching, such as the study of Emerson and Miyake (2003), made use of an articulatory suppression condition in which the participants are instructed either to say "a-b-c" aloud (cf. verbal secondary task condition) or to tap the foot (cf. motor secondary task condition). Emerson and Miyake (2003) found in their experiments that set-selection costs were only increased in the verbal and not in the motor secondary task. The authors conclude that this finding supports the view that the increase of set-selection costs is specific to the disruption of inner speech and is not due to a general increase of dual-task demands.

The findings of the present experiment are only partly consistent with their results. Similar to the Emerson and Miyake study, the younger adults indeed showed a greater increase in set-selection costs for the verbal than for the motor secondary task (see Fig. 3). In contrast, children and older adults showed a greater increase in set-selection costs for the motor secondary task as compared with the verbal secondary task. Hence, impairments of older adults and children in selecting between task sets are stronger when the interference occurs from the same output system. In the present case, the interference is greater when the competition during task preparation comes from the motor output system (involving the same effectors as in the two tasks A and B) than from the inner speech system. Note that in the present experiment both the motor and the verbal secondary task condition activate task-relevant knowledge, which is not the case in the study described above. Thus, when the processing of two tasks overlaps then set-selection costs increase when the same effectors have to be recruited for task execution. The findings are generally consistent with recent suggestions that deficits in task switching are primarily due to limitations in resolving interference at output stages in the elderly (e.g., Hartley $\&$ Little, 1999) or in inhibiting alternative response sets in children (e.g., Cepeda et al., 2001).

Interestingly, older adults compared with younger adults and children showed no increase in set-selection costs when the secondary task was a verbal task. This finding appears consistent with the view that inner speech is well-automatized during adulthood and a useful strategy to activate (retrieve) and/or maintain currently relevant task-set representations (cf. Emerson \& Miyake, 2003). Thus, the use of inner speech might serves as compensatory mechanisms to reduce impairments in executive functioning in the elderly. On the other hand, the use of inner speech is less practiced in children and therefore may cannot be used as well to compensate for executive deficits in action regulation.

Still an open question for future research is how the observed age differences in task switching will be affected when external task-set cues are absent. For instance, Emerson \& Miyake (2003) demonstrated that the increase in set-selection costs in the articulatory suppression condition is less pronounced when external cues help to activate and maintain the intended tasks, that is, age differences in the increase of set-selection costs may are much greater when no external cues are provided. 
The third goal of this study was to demonstrate whether facilitation and interference effects can be elicited by verbalizations of task-compatible and task-incompatible words. Our findings provide initial evidence that it is possible to differentially activate (or prime) response execution by verbalizations of intention-related knowledge at the level of response sets. Moreover, the results also indicated an age-differential pattern of facilitation and interference effects across the lifespan, that is, facilitation effects were more pronounced for children than for adults suggesting that children appear to strongly profit from task-compatible verbalizations for task execution than younger and older adults do. Unless this finding is not replicated we will discuss it only with caution because age differences in priming effects are known to be relatively unreliable (e.g., Bestgen \& Duport, 2000). However, consistent with a number of other findings is that interference effects were larger for older adults than for younger adults, even under single-task conditions when the other task is not required. This finding strongly supports the view that older adults have a major deficit in differentiating among potentially relevant task contents (Kray, 2004b; Mayr, 2001) as well as to disengage from no longer relevant task sets (Mayr \& Liebscher, 2001). Irrespective of the agedifferential effects in facilitation and interference effects, children and older adults clearly show greater priming effects than younger adults, supporting the general view that age changes go from more stimulus-driven behavior in childhood to more internally controlled behavior in young adulthood and then go back to more stimulus-driven behavior in old age (Belmont, 1996; Duncan, 1995; cf. Cepeda et al., 2001).

To summarize and conclude, the present results confirm the view that the age function in executive control components across the lifespan is not an uniform phenomenon: An inverted u-shaped age function was obtained for executive control components related to the selection of task sets. On the other hand, a negative linear age function was found for the coordination of task preparation and verbal processes. Although older adults generally benefited from verbalizations during task preparation, the children showed stronger facilitation of task execution by verbalization of compatible information. As a caveat, we note that the present findings are limited to a specific paradigm and specific stimulus materials, and are only based on three age groups and not on an age-continuous sample. Nevertheless they provide initial insights into the modulation of age-related changes in executive functioning through inner speech.

\section{Acknowledgements}

The present research was funded by the Deutsche Forschungsgemeinschaft (grant KR 18847/3-1). Special thanks go to Sandra Schappert, Babette Koch, and Julia Karbach for their help in recruiting participants and for running the experiments.

\section{References}

Baddeley, A. (1996). Exploring the central executive. The Quarterly Journal of Experimental Psychology, $49 A, 5-28$. 
Baddeley, A., Chincotta, D., \& Adlam, A. (2001). Working memory and the control of action: Evidence from task switching. Journal of Experimental Psychology: General, 130, 641-657.

Bedard, A.-C., Nichols, S., Barbosa, J. A., Schachar, R., Logan, G. D., \& Tannock, R. (2002). The development of selective inhibitory control across the life span. Developmental Neuropsychology, 21, 93-111.

Belmont, J. (1996). The "inverted U" shapedness of mental development. Journal of Russian and East European Psychology, 34, 12-15.

Bestgen, Y., \& Duport, V. (2000). Is negative priming a reliable measure for studying individual differences in inhibition. Cahiers de Psychologie/Current Psychology of Cognition, 19, 287-305.

Brinley, J. F. (1965). Cognitive sets, speed and accuracy of performance in the elderly. In A. T. Welford \& J. E. Birren (Eds.), Behavior, aging, and the nervous system (pp. 114-149). Springfield: Charles C. Thomas.

Case, R. (1992). The role of the prefrontal lobes in the regulation of cognitive development. Brain and Cognition, 20, 51-73.

Cepeda, N. J., Kramer, A. F., \& Gonzalez de Sather, J. C. M. (2001). Changes in executive control across the life span: Examination on task-switching performance. Developmental Psychology, 37, 715-730.

Comalli, P. E., Wapner, S., \& Werner, H. (1962). Interference effects of Stroop Color-Word Test in childhood, adulthood, and aging. Journal of Genetic Psychology, 100, 47-53.

De Jong, R. (2001). Adult age differences in goal activation and goal maintenance. European Journal of Cognitive Psychology, 13, 71-89.

Dempster, F. N. (1992). The rise and fall of the inhibitory mechanism: Toward a unified theory of cognitive development and aging. Developmental Review, 12, 45-75.

Diamond, A., \& Taylor, C. (1996). Development of an aspect of executive control: Development of the abilities to remember what I said and to "Do as I say, not as I do". Developmental Psychobiology, 29, 315-334.

Duncan, J. (1995). Attention, intelligence, and the frontal lobes. In M. S. Gazzaniga (Ed.), The cognitive neurosciences (pp. 721-733). Cambridge, MA: MIT Press.

Emerson, M. J., \& Miyake, A. (2003). The role of inner speech in task switching: A dual-task investigation. Journal of Memory and Language, 48, 148-168.

Frensch, P. A., Lindenberger, U., \& Kray, J. (1999). Imposing structure on an unstructured environment: Ontogenetic changes in the ability to form rules of behavior under conditions of low environmental predictability. In A. Friederici \& R. Menzel (Eds.), Learning: Rule extraction and representation (pp. 139-162). Berlin, Germany: De Gruyter.

Goschke, T. (2000). Intentional reconfiguration and involuntary persistence in task-set switching. In S. Monsell, \& J. Driver (Eds.), Control of cognitive processes: Attention and performance XVIII (pp. 331355). Cambridge MA.

Gruber, O., \& Goschke, T. (2004). Executive control emerging from dynamic interactions between brain systems mediating language, working memory and attentional processes. Acta Psychologica, 115, 105121 (this issue).

Hartley, A. A., \& Little, D. M. (1999). Age-related differences and similarities in dual-task interference. Journal of Experimental Psychology: General, 128, 416-449.

Heyder, K., Suchan, B., \& Daum, I. (2004). Cortico-subcortical contributions to executive control. Acta Psychologica, 115, 271-289 (this issue).

Kramer, A. F., Hahn, S., \& Gopher, D. (1999). Task coordination and aging: Explorations of executive control processes in the task switching paradigm. Acta Psychologica, 101, 339-378.

Kray, J. (2004a). Age-related changes in executive control: The nature of task-set cues in task preparation, in preparation.

Kray, J. (2004b). Selecting among ambiguous task sets in younger and older adults: The role of task cueing and item-specific learning, in preparation.

Kray, J., Li, K. Z. H., \& Lindenberger, U. (2002). Age-related changes in task-switching components: The role of task uncertainity. Brain and Cognition, 49, 363-381.

Kray, J., \& Lindenberger, U. (2000). Adult age differences in task switching. Psychology and Aging, 15, $126-147$. 
Li, S.-C., Lindenberger, U., Hommel, B., Aschersleben, G., Prinz, W., \& Baltes, P. B. (in press). Lifespan transformations in the couplings between intellectual abilities and constituent cognitive processes. Psychological Science.

Luria, A. R. (1969). Speech development and the formation of mental processes. In M. Cole \& I. Maltzman (Eds.), A handbook of contemporary Soviet psychology. New York: Basic Books.

Mayr, U. (2001). Age differences in the selection of mental sets: The role of inhibition, stimulus ambiguity, and response-set overlap. Psychology and Aging, 16, 96-109.

Mayr, U., Kliegl, R., \& Krampe, R. T. (1996). Sequential and coordinative processing dynamics in figural transformations across the life span. Cognition, 59, 61-90.

Mayr, U., \& Liebscher, T. (2001). Is there an age deficit in the selection of mental sets? European Journal of Cognitive Psychology, 13, 47-69.

Meacham, J. A. (1979). The role of verbal activity in remembering the goals of actions. In G. Zivin (Ed.), The development of self-regulation through private speech (vol. 6, pp. 237-263). New York: John Wiley \& Sons, Inc.

Meiran, N. (1996). Reconfiguration of processing mode prior to task performance. Journal of Experimental Psychology: Learning, Memory, and Cognition, 22, 1423-1442.

Meiran, N., Gotler, A., \& Perlman, A. (2001). Old age is associated with a pattern of relatively intact and relatively impaired task-set switching abilities. Journals of Gerontology: Psychological Sciences and Social Sciences, 56, 88-102.

Milham, M. P., Erickson, K. I., Banich, M. T., Kramer, A. F., Webb, A., Wszalek, T., \& Cohen, N. J. (2002). Attentional control in the aging brain: Insights from an fMRI study of the Stroop task. Brain and Cognition, 49, 277-296.

Miller, E. K., \& Cohen, J. D. (2001). An integrative theory of prefrontal cortex function. Annual Review of Neuroscience, 24, 167-202.

Miyake, A., Emerson, M. J., Padilla, F., \& Ahn, J.-c. (2004). Inner speech as a retrieval aid for task goals: the effects of cue type and articulatory suppression in the random task cuing paradigm. Acta Psychologica, 115, 123-142 (this issue).

Miyake, A., Friedman, N. P., Emerson, M. J., Witzki, A. H., Howerter, A., \& Wager, T. D. (2000). The unity and diversity of executive functions and their contributions to a complex frontal lobe task: A latent variable analysis. Cognitive Psychology, 41, 49-100.

Morin, A., \& Everett, J. (1990). Inner speech as a mediator of self-awareness, self-consciousness and selfknowledge: A hypothesis. New Ideas in Psychology, 8, 337-356.

Prull, M. W., Gabrieli, J. D. E., \& Bunge, S. A. (2000). Age-related changes in memory: A cognitive neuroscience perspective. In F. I. M. Craik \& T. A. Salthouse (Eds.), Handbook of aging and cognition (pp. 91-154). Mahwah, NJ: Lawrence Erlbaum.

Raz, N. (2000). Aging of the brain and its impact on cognitive performance: Integration of structural and functional findings. In F. I. M. Craik \& T. A. Salthouse (Eds.), Handbook of aging and cognition (2nd ed., pp. 1-90). Mahwah, NJ: Lawrence.

Roberts, R. J., \& Pennington, B. F. (1996). An interactive framework for examining prefrontal cognitive processes. Developmental Neuropsychology, 12, 105-126.

Rogers, R. D., \& Monsell, S. (1995). Costs of a predictable switch between simple cognitive tasks. Journal of Experimental Psychology: General, 124, 207-231.

Shallice, T., \& Burgess, P. (1993). Supervisory control of action and thought selection. In A. Baddeley \& L. Weiskrantz (Eds.), Attention: Selection, awareness, and control (pp. 171-187). Oxford: Clarendon Press.

Vygotsky, L. S. (1988). On inner speech. In M. B. Franklin \& S. Barten (Eds.), Child language: A reader (pp. 181-187). New York: Oxford University Press.

West, R. L. (1996). An application of prefrontal cortex function theory to cognitive aging. Psychological Bulletin, 120, 272-292.

Zelazo, P. D. (1999). Self-reflection and the development of consciously controlled processing. In P. Mitchell \& K. J. Riggs (Eds.), Children's reasoning and the mind (p. 5). Hove, UK: Psychology Press.

Zelazo, P. D., Craik, F. I. M., \& Booth, L. (2004). Executive function across the life span. Acta Psychologica, 115, 167-183 (this issue). 